EPJ Web of Conferences 36, 00019 (2012)

DOI: $10.1051 /$ epjconf/20123600019

(C) Owned by the authors, published by EDP Sciences, 2012

\title{
Resonance studies with the UrQMD model
}

\author{
Sascha Vogel ${ }^{1, a}$, Marcus Bleicher ${ }^{2}$ \\ ${ }^{1}$ Frankfurt Institute for Advanced Studies, Ruth-Moufang-Str. 1, 60438 Frankfurt, Germany \\ ${ }^{2}$ SUBATECH, Laboratoire de Physique Subatomique et des Technologies Associées \\ University of Nantes - IN2P3/CNRS - Ecole des Mines de Nantes \\ 4 rue Alfred Kastler, F-44072 Nantes Cedex 03, France
}

\begin{abstract}
These proceedings will cover various studies of hadronic resonances within the UrQMD transport model. After a brief explanation of the model, various observables will be highlighted and the chances for resonance reconstruction in hadronic channels will be discussed. Possible signals of chiral symmetry restoration will be investigated for feasibility.
\end{abstract}

\section{Introduction}

The exploration of hot and dense matter created in heavy ion collision is one of the most complex challenges in present day physics. The initial state is unknown, although speculations at high energies exist [1-4]. The proper theoretical treatment of interactions is still heavily debated (see e.g.[5,6]) and the transition from a possibly deconfined phase to a hadronic phase is quite poorly understood. Resonances provide an unique approach to learn about the hot and dense phase which is produced in heavy ion collisions [7-25]. Since most hadronic resonances decay on time scales of several $\mathrm{fm} / \mathrm{c}$, plenty of short-lived resonances will decay within the created medium and will carry the information from that stage of the collision. This is of course dependent on the kinematics of the particles and will be discussed later in detail.

These proceedings are structured as follows: After a brief introduction to the theoretical model in Section 2 the possibility to explore the dense phase of heavy ion collisions using hadronic decay channels of resonances will be discussed in Section 3. The effects of baryons will be presented and explained in Section 4. In Section 5 the possibility of measuring the chiral partner of the $\rho$ vector meson, the $a_{1}$ meson, will be discussed. The manuscript will end with conclusions.

\section{Model description}

For the calculations discussed in these proceedings the UrQMD model has been used, a non-equilibrium transport approach, which relies on the covariant Boltzmann equation. All cross sections are calculated by the principle of detailed balance and the additive quark model or are fitted to available data. UrQMD does not include any explicit in-medium modifications for vector mesons or effects to describe the restoration of chiral symmetry. The model allows to study the full space time evolution of all hadrons, resonances and their decay products in hadron-hadron or nucleus-nucleus collisions. This permits to explore the emission patterns of resonances in detail and to gain insight into their origins and decay channels. For previous studies of resonances within this model see [14-23]. For further details about

a e-mail: svogel@th.physik.uni-frankfurt.de

This is an Open Access article distributed under the terms of the Creative Commons Attribution License 2.0, which permits unrestricted use, distribution, and reproduction in any medium, provided the original work is properly cited. 
the UrQMD model the reader is referred to $[26,27]$. Recent development concerning the inclusion of a hydrodynamic phase are described in [28].

Experimentally, the reconstruction of resonances is challenging. One often applied technique is to reconstruct the invariant mass spectrum for single events. Then, an invariant mass distribution of mixed events is generated (here, the particle pairs are uncorrelated by definition). The mixed event distribution is subtracted from the invariant mass spectrum of the single (correlated) events. As a result one obtains the mass distributions and yields (after all experimental corrections) of the resonances by fitting the resulting distribution with a suitable function (usually a Breit-Wigner function peaked around the pole mass of the respective resonance).

If the resonance spectral function changes in the hadronic medium this is in principle visible in the difference spectrum between true and mixed events. However, if a daughter particle (re-)scatters before reaching the detector the signal for the experimental reconstruction is blurred or even lost. Especially for strongly interacting decay products this effect can be sizeable. It is therefore difficult to judge whether a deviation from an expected Breit-Wigner distribution is due to an initial deformation or an increase of the initial width or due to the momentum dependence of the rescattering cross section of the daughter particles.

What makes this analysis even tougher is the fact that the resonances decay over a wide range of densities and therefore only an average value is measured. If this average value is dominated by resonance decays at low density the information from the high density phase is blurred and may offer only a limited view on the high density phase of the heavy ion collision.

UrQMD offers a different technique for the extraction of resonances which we apply here. We follow the individual decay products of each decaying resonance (the daughter particles). If the daughter particles do not rescatter in the further evolution of the system, the resonance is counted as 'reconstructable'. The advantage of this method is that it allows to trace back the origin of each individual resonance to study their spatial and temporal emission pattern. Because UrQMD follows the space time evolution of all particles it is possible to link production and decay point of each individual resonance. This method also allows to explore the reconstruction efficiency in different decay branches.

In order to calculate at which density the resonance decays we have to determine the baryonic density. The baryon density is calculated locally at the position of the resonance in the rest frame of the baryon current (Eckart frame) as $\rho_{B}=j^{0}$ with $j^{\mu}=\left(\rho_{B}, \mathbf{0}\right)$. Details on the calculation of the baryon density are discussed in [20]. In all figures we present the density in units of ground state density, where a value of $0.161 / \mathrm{fm}^{3}$ is assumed.

\section{Density distribution of reconstructable resonances}

Complementary to the study of bulk matter created in ultra-relativistic heavy ion collisions is the measurement and theoretical analysis of rare probes, such as charmed particles, dileptons and resonances. Hadronic resonances decay on time scales of several $\mathrm{fm} / \mathrm{c}$, which is well within the expected time scales of the developing medium in heavy ion collisions, even after taking into account resonance kinematics [29]. Within a hot and dense medium resonances, most notably vector mesons like the $\rho$ meson are supposed to change their spectral function [30-32], however in order to disentangle interesting effects like e.g. the restoration of chiral symmetry from trivial effects it is crucial to understand the kinematics of the resonances and the surrounding medium. Due to space constraints the focus in this paper is put on the FAIR energy regime, however all caveats apply up to RHIC (and possibly even up to LHC) energies as well.

Before discussing the effect of any medium-modifications due to a surrounding high density medium it is useful to check the density distribution of decaying resonances. Shown in Fig. 1 (left) is the probability distribution of baryon density at the point of production for various mesonic and baryonic resonances. The general trend shows that reconstructable resonances are produced at very low baryon densities, which is naively expected. What is notable is the fact that most reconstructable resonances decay completely outside the medium. Another way to look at this is to ask a slightly different question. Depicted in Fig. 1 (right) is the probability to reconstruct a certain resonance species as a function of 

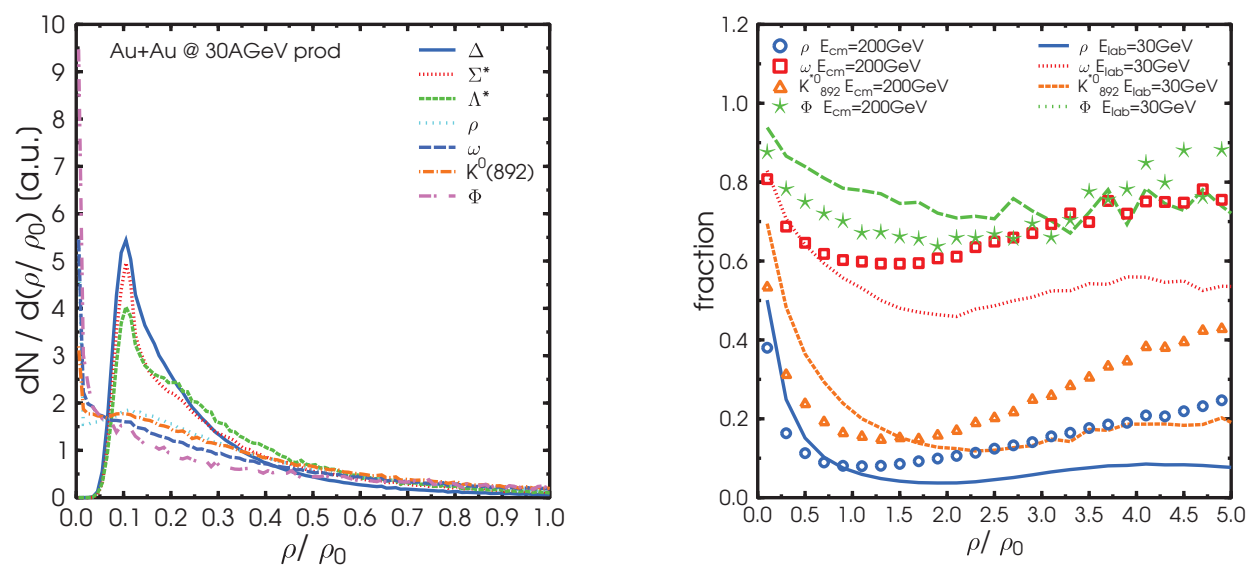

Fig. 1. (Color online) (Left) Probability distribution of baryon density at the production vertex for various reconstructable resonances in central $(\mathrm{b} \leq 3.4 \mathrm{fm}) \mathrm{Au}+\mathrm{Au}$ collisions at $30 \mathrm{AGeV}$ as a function of baryon density. One observes that most resonances which can be reconstructed in the hadronic decay channel originate from low baryon density. (Right) Fraction of reconstructable meson resonances as a function of baryon density at the point of production. Baryons resonances are not shown, however exhibit the same qualitative behaviour.

baryon density. As naively expected the probability is highest at very low densities and decreases with increasing density. A surprising feature shows up at roughly $1.5-2 \rho_{0}$, where the 'reconstructability' increases again. This feature originates from the fact that resonances produced at high baryon density on average have a high transverse momentum which enables the decay products to escape the collision zone undisturbed. Fig. 2 (left), which depicts the average transverse momentum of $\Delta$ resonances as a function of baryon density strengthens this argumentation. Lines show reconstructable resonances, symbols show all decayed resonances. The striking feature is the different average transverse momentum between all decayed resonances and those which are reconstructable. The higher the average transverse momentum, the larger is the chance that the resonance can be reconstructed. The $\left\langle p_{T}>\right.$ of reconstructable $\Delta$ resonances is about $200 \mathrm{MeV}$ higher than for all $\Delta$ resonances. Resonances with a large $p_{T}$ can leave the high density zone rather fast and move with a velocity of about $\left\langle p_{T}>/ m\right.$ outwards. Although only $\Delta$ baryons are shown it has been checked that this feature is independent of resonance species.

Another interesting feature in Fig. 2 (left) is the difference between the $\sqrt{s}=200 \mathrm{AGeV}$ and $E_{l a b}=30 \mathrm{AGeV}$ curves. While the $E_{l a b}=30 \mathrm{AGeV}$ data shows a decrease of $<p_{T}>$ as a function of the baryon density, the $\sqrt{s}=200 \mathrm{AGeV}$ data show an increase. At $\sqrt{s}=200 \mathrm{AGeV}$ the initial collisions (which happen at high baryon density) are more energetic and give the particles a high transverse momentum, subsequent rescattering decreases $p_{T}$. For the $E_{l a b}=30 \mathrm{AGeV}$ collisions the situation is opposite. Initially the particle $p_{T}$ is small and the rescattering increases the $p_{T}$ due to transverse expansion. This effect depends on the collision energy and the initial transverse momentum the produced particles pick up. More details can be found in [22]

Depicted in Fig. 2 (right) is the total transverse momentum spectrum for $\Delta, \Lambda, \Sigma$ baryons, as well as $\rho, \omega, K^{* 0}$ and $\omega$ mesons, with full circles depicting the spectrum for all decayed resonances and open circles for reconstructable ones. The numbers in the shaded areas indicate the percentage of reconstructable resonances stemming from density region with $\rho / \rho_{0}>2$. One observes a clear correlation between transverse momentum and density. For more information, please refer to [22]. 

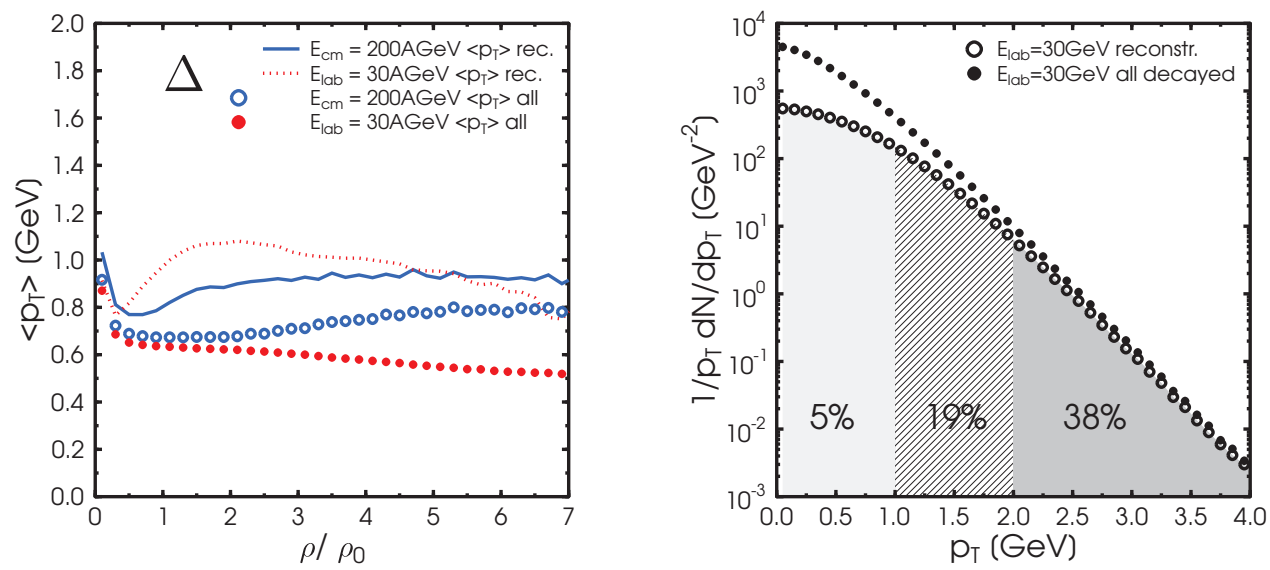

Fig. 2. (Color online) (Left) Average transverse momentum of reconstructable (line) or all (symbol) $\Delta$ baryons as a function of baryon density for two different energies. (Right) Transverse momentum spectra for all and reconstructable resonances for central $(\mathrm{b} \leq 3.4 \mathrm{fm}) \mathrm{Au}+\mathrm{Au}$ collision at $30 \mathrm{AGeV}$ beam energy. Full circles depict the spectrum for all decayed resonances (included in the analysis are $\Delta, \Lambda, \Sigma$ baryons, as well as $\rho, \omega, K^{* 0}$ and $\omega$ mesons), open circles for reconstructable resonances. The numbers indicate the percentage of reconstructable resonances stemming from density region with $\rho / \rho_{0}>2$.

\section{The effect of baryon kinematics}

When analyzing vector meson mass shifts it is crucial to have a proper baseline to compare to. At certain energy scales mass shifts might have rather trivial reasons, such as the decay kinematics of baryons. Shown in Fig. 3 (left) is the mass spectrum of $\rho$ mesons as obtained from $\mathrm{C}+\mathrm{C}$ collisions at $2 \mathrm{AGeV}$. Due to the decay of the $\mathrm{N}^{*}(1520)$ resonance a strong bias towards low masses is given, which originates from the fact that the $\mathrm{N}^{*}(1520)$ decays to a large percentage into the $\mathrm{N} \rho$ channel (roughly $20 \%$, see [33]). There is a strong kinematic bound on the respective $\rho$ mass in this decay channel. Since the nucleon has a fixed mass of $938 \mathrm{MeV}$, energy conservation dictates a low mass peak of the decay $\rho \mathrm{s}$ at around 500-600 MeV. This back-of-the-envelope estimate $(1520 \mathrm{MeV}-938 \mathrm{MeV}=582 \mathrm{MeV})$ of course does not take into account that both the decaying baryon resonance and the daughter-particle have a width, which of course needs to be taken into account in the calculations.

The aforementioned effect distorts the $\rho$ meson mass spectrum, with the effect being essentially independent of the collision system. The major factor is the collision energy and the resulting baryon / meson ratio. The more dominant the $\pi \pi \rightarrow \rho$ production channel becomes, the less prominent the low mass tail becomes.

Another bias which distorts $\rho$ meson mass spectra is the rapidity dependence of the baryon / meson ratio. Shown in Fig. 3 (right) is the average mass of $\rho$ mesons in $\mathrm{C}+\mathrm{C}$ collisions at $2 \mathrm{AGeV}$ (blue, lower line) and in $\mathrm{Pb}+\mathrm{Pb}$ collisions at $30 \mathrm{AGeV}$ (red, upper line). One observes that the average $\rho$ meson mass decreases as a function of rapidity. The reason for this is the aforementioned effect. The higher the baryon content, the more prominent the low mass tail is and the lower the average $\rho$ meson mass becomes. Thus, when measuring away from mid-rapidity, the average mass $\rho$ meson mass will drop, independent of any hot and dense matter effects, such as the potential restoration of chiral symmetry. 

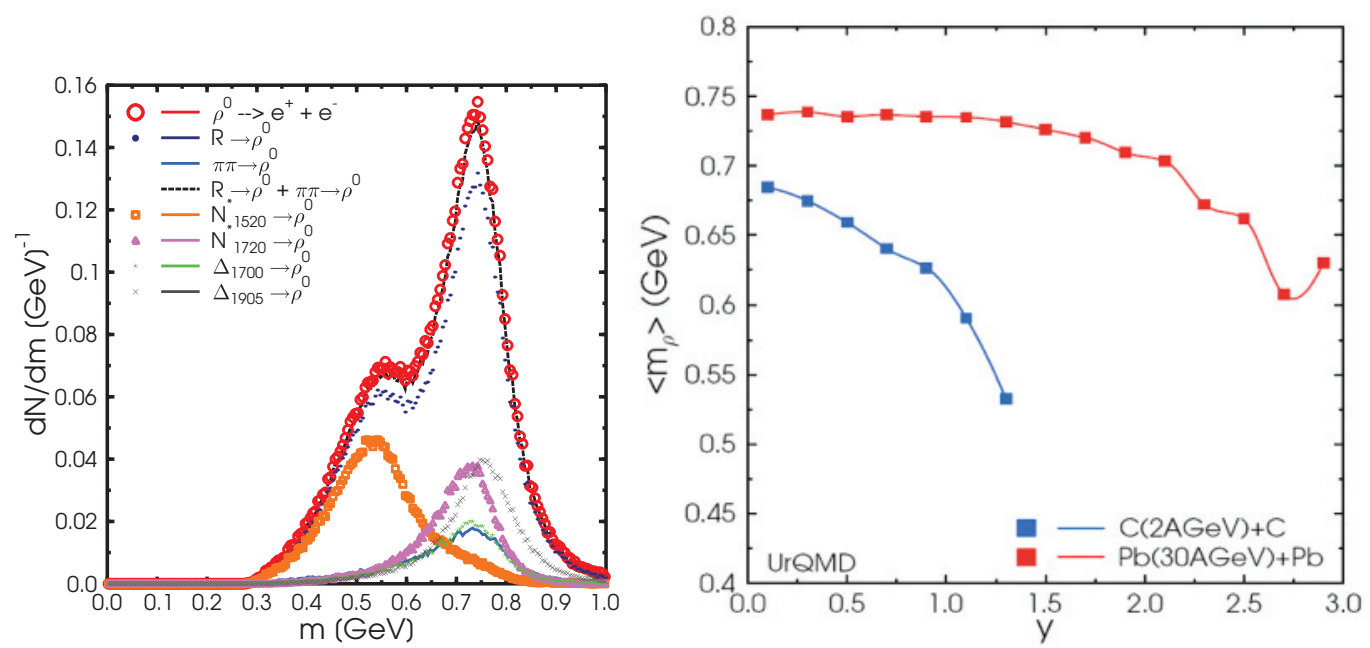

Fig. 3. (Color online) (Left) Mass spectrum of $\rho$ mesons with a detailed analysis of the different production channels. $\rho$ mesons produced by $N^{*}(1520)$ decays produce a low mass bump in the total spectrum. (Right) Average $\rho$ meson mass as a function of rapidity.

\section{$5 a_{1}$ meson kinematics}

A clear signal for the detection of the chirally restored phase is the measurement of the $a_{1}$ meson spectral function or respectively the mass spectrum and then compare it to the $\rho$ meson spectral function. While measuring the elusive $a_{1}$ meson is a feat in itself, the decay kinematics make the measurement even more difficult. When analyzing the up to date accessible particle information [33] on the $a_{1}$ it becomes clear that realistically only 2 decay channels are feasible to analzyse in experimental setups, namely $a_{1} \rightarrow \rho \pi$ and $a_{1} \rightarrow \gamma \pi$. The $a_{1} \rightarrow \rho \pi$ decay is quite challenging to measure, since it involves three-particle correlations, however the $a_{1} \rightarrow \gamma \pi$ channel has its own caveats as will be discussed in the following. For the full discussion the reader is referred to [19].

As shown in Fig. 4 (left) the $a_{1}$ meson branching ratio is strongly mass dependent, with a strong bias towards the $\gamma \pi$ channel at low masses and a suppression of that channel at higher masses. In order get a feeling for the relevant mass scales a Breit-Wigner distribution centered around the $a_{1}$ pole mass of $1260 \mathrm{MeV}$ is plotted as a shaded area. The dashed-dotted line depicts the $a_{1}$ meson mass spectrum as obtained from UrQMD calculations. Both the Breit-Wigner distribution and the mass spectrum are normalised for better comparison. By folding the branching ratio of the $a_{1} \rightarrow \gamma \pi$ channel with the full mass spectrum one obtains the mass spectrum seen in that channel. Due to the strong mass dependence in the branching ratio the mass spectrum seen in a specific channel will not necessarily look like a 'trivial' Breit-Wigner-resonance-distribution. Shown in Fig. 4 (right) is the mass spectrum as obtained from the $\gamma \pi$ channel. One observes a clear double peak structure with one peak centered roughly around the nominal $a_{1}$ meson mass and one peak at much lower masses at around $500 \mathrm{MeV}$. This is exactly the mass region where one would expect the $\rho$ meson in heavy ion collisions, thus making a comparison of mass spectra of both mesons very hard to nigh impossible.

Future experiments like the CBM experiment at FAIR might have a chance to analyze the $\rho \pi$ channel via $e^{+} e^{-} \pi$ correlations, however further studies are needed to check the feasibility of this measurement.

\section{Conclusions}

The measurement and theoretical understanding of hadronic resonances in heavy ion collisions proves to be difficult and do not necessarily reflect the hot and dense stage of heavy ion collisions. Experimentally reconstructable resonances originate rarely from a stage of high baryon density, by applying a $p_{T}$ 

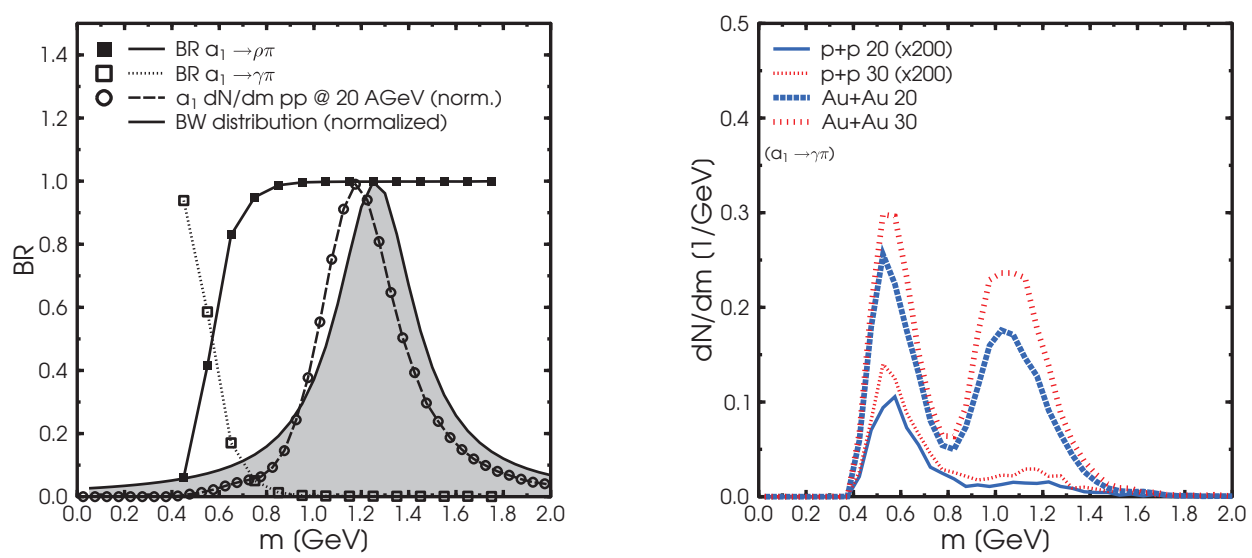

Fig. 4. (Color online) (Left) Mass dependent branching ratios for the $a_{1}$ meson with the two exit channel of $\gamma \pi$ and $\rho \pi$ as calculated from UrQMD. Filled squares depict the branching ratio of $a_{1}$ mesons into the exit channel $\rho \pi$, whereas open squares depict the branching ratio into $\gamma \pi$. Below a mass of $750 \mathrm{MeV}$ the decay channel $a_{1} \rightarrow \rho \pi$ is kinematically suppressed and the channel $a_{1} \rightarrow \gamma \pi$ dominates. At masses above $750 \mathrm{MeV}$ the branching ratio into $\rho \pi$ increases steeply. The grey shaded area depicts a normalised Breit-Wigner distribution around the $a_{1}$ pole mass, whereas the circles depict the normalised mass spectrum of the $a_{1}$ meson as obtained from UrQMD calculations for $\mathrm{p}+\mathrm{p}$ collisions at $20 \mathrm{AGeV}$. (Right) Mass distribution of al mesons which can be reconstructed in $\gamma \pi$ correlations in nucleus-nucleus and proton- proton collisions at 20 and $30 \mathrm{AGeV}$. Note that the $\mathrm{p}+\mathrm{p}$ curves have been multiplied by 200 for visibility.

cut one can increase the fraction of reconstructable resonances from the dense stage of the collision. Baryon decay kinematics distort the mass spectrum, especially at low beam energies. This effect vanishes at higher beam energies, since the production channel of $\rho$ mesons shifts from baryonic resonances to $\pi \pi$ scattering. The average $\rho$ meson mass is additionally dependent on rapidity, which needs to be considered when doing the experimental analysis.

While the measurement of the $a_{1}$ meson still proves to be a prominent probe for the investigation of chiral symmetry, the $a_{1} \rightarrow \gamma \pi$ decay channel might make a measurement and a proper interpretation next to impossible. Mass-dependent branching ratios produce a bias at low masses, right in the mass region of the $\rho$ meson mass.

\section{Acknowledgements}

The computational resources have been provided by the Center for Scientific Computing (CSC) at the Goethe-University of Frankfurt. This work was supported by GSI, BMBF and the Hessian LOEWE initiative through the Helmholtz International Center for FAIR (HIC for FAIR).

\section{References}

1. E. Iancu, A. Leonidov and L. D. McLerran, Nucl. Phys. A 692 (2001) 583

2. E. Ferreiro, E. Iancu, A. Leonidov and L. McLerran, Nucl. Phys. A 703 (2002) 489

3. M. Gyulassy and L. McLerran, Nucl. Phys. A 750 (2005) 30

4. A. Dumitru, F. Gelis, L. McLerran and R. Venugopalan, Nucl. Phys. A 810 (2008) 91

5. W. A. Horowitz and M. Gyulassy, Phys. Lett. B 666 (2008) 320 
6. W. A. Horowitz, AIP Conf. Proc. 1441 (2012) 889

7. G. Agakichiev et al. [HADES], Phys. Rev. Lett. 98, 052302 (2007)

8. X. Lopez et al., Phys. Rev. C 76, 052203 (2007).

9. S. V. Afanasev et al. [NA49], J. Phys. G 27, 367 (2001).

10. D. Adamova et al. [CERES], Phys. Rev. Lett. 91, 042301 (2003)

11. J. Adams et al. [STAR], Phys. Rev. Lett. 97, 132301 (2006)

12. B. I. Abelev et al. [STAR], Phys. Rev. C 78, 044906 (2008)

13. P. Fachini, J. Phys. G 35, 044032 (2008).

14. M. Bleicher and J. Aichelin, Phys. Lett. B 530 (2002) 81

15. M. Bleicher, Nucl. Phys. A 715 (2003) 85

16. M. Bleicher and H. Stoecker, J. Phys. G G 30 (2004) S111

17. S. Vogel and M. Bleicher, Phys. Rev. C 74 (2006) 014902

18. D. Schumacher, S. Vogel and M. Bleicher, Acta Phys. Hung. A 27 (2006) 451

19. S. Vogel and M. Bleicher, Phys. Rev. C 78 (2008) 064910

20. S. Vogel, H. Petersen, K. Schmidt, E. Santini, C. Sturm, J. Aichelin and M. Bleicher, Phys. Rev. C 78 (2008) 044909

21. K. Schmidt, E. Santini, S. Vogel, C. Sturm, M. Bleicher and H. Stocker, Phys. Rev. C 79 (2009) 064908

22. S. Vogel, J. Aichelin and M. Bleicher, Phys. Rev. C 82 (2010) 014907

23. S. Vogel, J. Aichelin and M. Bleicher, J. Phys. G G 37 (2010) 094046

24. G. Torrieri, S. Steinke, W. Broniowski, W. Florkowski, J. Letessier and J. Rafelski, Comput. Phys. Commun. 167 (2005) 229

25. G. Torrieri, S. Jeon, J. Letessier and J. Rafelski, Comput. Phys. Commun. 175 (2006) 635

26. S. A. Bass, M. Belkacem, M. Bleicher, M. Brandstetter, L. Bravina, C. Ernst, L. Gerland and M. Hofmann et al., Prog. Part. Nucl. Phys. 41 (1998) 255 [Prog. Part. Nucl. Phys. 41 (1998) 225]

27. M. Bleicher, E. Zabrodin, C. Spieles, S. A. Bass, C. Ernst, S. Soff, L. Bravina and M. Belkacem et al., J. Phys. G G 25 (1999) 1859

28. H. Petersen, J. Steinheimer, G. Burau, M. Bleicher and H. Stocker, Phys. Rev. C 78 (2008) 044901

29. C. Markert, R. Bellwied and I. Vitev, Phys. Lett. B 669 (2008) 92

30. R. Rapp and J. Wambach, Adv. Nucl. Phys. 25 (2000) 1

31. H. van Hees and R. Rapp, Phys. Rev. Lett. 97 (2006) 102301

32. H. van Hees and R. Rapp, Nucl. Phys. A 806 (2008) 339

33. C. Amsler et al. [Particle Data Group Collaboration], Phys. Lett. B 667 (2008) 1. 